\section{2d IMPROVING AWARENESS OF NANOMATERIALS AMONG U.S. CONSTRUCTION WORKERS}

BE Lippy, GH West. The Centre for Construction Research and Training, Silver Spring, MD, USA

\subsection{6/oemed-2018-ICOHabstracts.203}

Introduction The Centre for Construction Research and Training (CPWR) has support from NIOSH to research the use of nano-enabled construction materials in the U.S. The Centre maintains a web-based inventory of $550+$ products believed to nano-enabled. The extent to which nano-enabled construction products have been commercialised remains unclear, however, because no U.S. regulation requires manufacturers to identify engineered nanoparticles (ENPs\} on labels or Safety Data Sheets (SDSs\}. Under U.S. OSHA's Hazard Communication Standard, manufacturers of nano-enabled products do not have to identify any component that represents less than one percent of the mix on a weight basis. Given the low mass of ENPs, OSHA's threshold may not be reached, allowing manufacturers to legally withhold information about nanoparticles. In 2008 at an EPA conference, the author recommended that OSHA require all ENPs to be identified on SDSs and that conditional language be included advising against using PELs for the parent material. Lee, et al. made similar recommendations about use of PELs in 2013 after evaluating 97 SDSs. They reported that $85 \%$ did not provide any nanomaterialspecific data. These authors developed a 2012 ISO technical report (ISO/TR 13329) that provides excellent guidance on preparing SDSs including identifying the nanoform components.

Methods In 2013-2014 CPWR surveyed 79 experienced construction tradespersons with an 11-item written survey. The instrument was designed to gauge perceptions and level of knowledge relating to use of nanotechnology in the construction industry. The survey protocol was approved by CPWR's Institutional Review Board prior to use.

Results Survey participants from various locations across the U.S. self-identified with 22 different construction trades. Masons, plasterers, and carpenters represented the majority of those surveyed (58\%). On average, the group reported having $30.5 \pm 9.4$ years of trade experience and $13.3 \pm 7.8$ years of training experience. Less than half of respondents (48\%) were aware that construction products containing nanomaterials are commercially available in the USA, and only $13 \%$ knew of a construction nanomaterial being used on an actual construction job site.

Conclusion This survey of construction health and safety trainers suggests that much more needs to be done to increase awareness of nanotechnology in the construction industry. Better risk communication and dissemination strategies focused on workers are needed. This isn't an American phenomenon. Broekhuizen, et al. found even higher levels of ignorance of nanomaterials among European construction workers in surveys conducted in 2009. Positive trends can be seen, however. The European Trade Union Institute has developed training materials for workers. CPWR is providing awareness training to union apprentice instructors and developing toolbox talks aimed at specific trades. Some manufacturers have begun to develop Health Product Declarations for their nano material products. These innovative hazard communication tools may address the well-documented inadequacies of Safety Data Sheets.

\section{2e UTILISING THE OSHWIKI: LESSONS LEARNED FOR DISSEMINATION AND ENGAGEMENT. THE EU-OSHA EXPERIENCE}

D Elsler. European Agency for Safety and Health at Work, Bilbao, Spain

10.1136/oemed-2018-ICOHabstracts.204

Introduction The European Agency for Safety and Health at Work (EU-OSHA) launched OSHwiki in August 2014 with the aim of being an authoritative source of information that supports government, policy-makers, employers' organisations and workers' representatives around Europe on all aspects of workplace health and safety.

Methods OSHwiki is a dynamic multilingual collaborative web platform based on the wiki concept providing users a new way of networking with the global OSH community and allowing them to collaborate in creating and sharing knowledge on occupational safety and health. It assures quality by restricting authorship to nominated members of the $\mathrm{OSH}$ community, providing those authors with recognition of their knowledge.

Results Following a feasibility study carried out to assess the advantages and drawbacks of OSHwiki, the results have shown that the current format does not realise its full potential. The strengths are primarily associated with the high degree of reliability of the OSHwiki content. However the main weaknesses are associated with the low visibility of OSHwiki in the OSH Community.

Conclusions By using this approach, EU OSHA has been able to maintain current information online on a wide spectrum of $\mathrm{OSH}$ topics in a number of different languages and is now looking at how to fulfil its full potential through Wikipedia.

\section{$1682 f$ PARTNERSHIPS TO EXPAND OCCUPATIONAL SAFETY AND HEALTH CONTENT IN WIKIPEDIA}

${ }^{1}$ T Morata, ${ }^{2} \mathrm{M}$ Lum. 'National Institute for Occupational Safety and Health, Cincinnati, USA; ${ }^{2}$ National Institute for Occupational Safety and Health, Washington DC, USA

\subsection{6/oemed-2018-ICOHabstracts.205}

Introduction Wikipedia is accessed by hundreds of millions around the world and that makes Wikipedia one of the most powerful platforms for the dissemination of science information. While Wikipedia offers high-quality content about certain topics, a large proportion of articles are insufficiently developed. The Wikimedia Foundation has engaged in partnerships with scientific and academic institutions to improve the coverage and communication of science to the public. These efforts are beneficial to professional and academic associations interested in sharing reliable, vetted information about their discipline with the world.

Methods The U.S. National Institute for Occupational Safety and Health (NIOSH) is one of the research agencies engaged in this effort. NIOSH developed and manages the WikiProject Occupational Safety and Health. NIOSH also participates in a classroom program (where students write Wikipedia articles) to expand and improve Wikipedia's content on public and occupational health. The evaluation of their efforts is conducted using an Altmetrics platform that tracks alternative metrics for information products located on the web that have a persistent tracking number, such as an ISBN or DOI. 